\title{
Tool Remaining Useful Life Prediction Using Deep Transfer Reinforcement Learning Based on Long Short Term Memory Networks
}

Jiachen Yao ( $D$ 791344334@qq.com )

Nanjing University of Science and Technology https://orcid.org/0000-0002-1575-891X

Baochun Lu

Nanjing University of Science and Technology

Junli Zhang

Nanjing University of Science and Technology

\section{Research Article}

Keywords: Tool wear monitoring, remaining useful life (RUL) prediction, deep reinforcement learning, transfer learning

Posted Date: March 31st, 2021

DOl: https://doi.org/10.21203/rs.3.rs-352693/v1

License: (c) (i) This work is licensed under a Creative Commons Attribution 4.0 International License. Read Full License

Version of Record: A version of this preprint was published at The International Journal of Advanced Manufacturing Technology on September 13th, 2021. See the published version at https://doi.org/10.1007/s00170-021-07950-2. 


\section{Author information}

Jiachen Yao

Nanjing University of Science and Technology, Nanjing, China

Email: 791344334@qq.com

Baochun Lu

Nanjing University of Science and Technology, Nanjing, China

Email: nlglbc@126.com

Junli Zhang

Nanjing University of Science and Technology, Nanjing, China

Email: maysevenhero@126.com

\section{Declarations}

\section{Ethical Approval}

NA.

\section{Consent to Participate}

NA.

\section{Consent to Publish}

Written informed consent for publication was obtained from all participants.

\section{Authors Contributions}

Jiachen Yao performed the data analyses and wrote the manuscript;

Baochun Lu contributed to the conception of the study;

Junli Zhang performed the experiment and helped perform the analysis.

\section{Funding}

This work was supported in part by the National Key R\&D Program of China under Grant 2018YFB1308300.

\section{Competing Interests}

The authors declare that they have no conflict of interest.

\section{Availability of data and materials}

The data sets supporting the results of this article are included within the article. 


\title{
Tool Remaining Useful Life Prediction Using Deep Transfer Reinforcement Learning Based on Long Short Term Memory Networks
}

\author{
Jiachen Yao, Baochun Lu, and Junli Zhang
}

\begin{abstract}
Tool wear and faults will affect the quality of machined workpiece and damage the continuity of manufacturing. The accurate prediction of remaining useful life (RUL) is significant to guarantee processing quality and improve productivity of automatic system. At present, the most methods for tool RUL prediction are trained by history fault data. However, when researching on new types of tools or processing high value parts, fault datasets are difficult to acquired, which led to RUL prediction a challenge under limited fault data. To overcome shortcomings of above prediction methods, a deep transfer reinforcement learning (DTRL) network based on long short term memory (LSTM) network is presented in this paper. Local features are extracted from consecutive sensor data to track the tool states, and the trained network size can be dynamically adjusted by controlling time sequence length. Then in DTRL network, LSTM network is employed to construct the value function approximation for smoothly processing temporal information and mining long-term dependencies. On this basis, a novel strategies of Q-function update and transfer are presented to transfer the DRL network trained by historical fault data to a new tool for RUL prediction. Finally, tool wear experiments are performed to validate effectiveness of the DTRL model. The prediction result demonstrate that the proposed method has high accuracy and generalization for similar tools and cutting conditions.
\end{abstract}

\section{Keywords}

Tool wear monitoring, remaining useful life (RUL) prediction, deep reinforcement learning, transfer learning

\section{Introduction}

Tool is a key part in manufacturing process, including turning, milling, cutting and so on. Tool wear in manufacturing process affects the machining performance and reduces the productivity of high-speed computer numerical control (CNC). Thus, effective tool wear monitoring and remaining useful life (RUL) prediction are of great significance for improving machining quality and predictive maintenance [1-3]. Generally, tool monitoring and RUL prediction methods can be roughly classified into statistical model-based and data-driven methods.

In the statistical model-based methods, the core idea is to establish a failure mechanisms model for RUL prediction on the basis of stochastic process. Si et al. [4] developed a Wiener-process-based degradation model for RUL prediction, and recursive filter was used to reduce the estimation error. Yan et al. [5] designed a stage-based Gamma process to predict the probability density function of tool unobservable degradation. Wang et al. [6] presented a particle filtering method for tool wear state prediction.

In the data-driven methods, machine and deep learning approaches are used to process observation data for diagnosis and prognosis [7]. In this kind of method, vibration sensors, torque sensors, or other kinds of sensors are installed on machining centers to monitor tool working states. Sensory signals are extracted by signal processing technology to get discriminant signal features [8-10]. Due to the advantages of high prediction accuracy and easy modeling, data-driven methods have been a research hotspot for tool state monitoring and RUL prediction. For instance, Widodo et al. [11] reviewed the implement of support vector machine (SVM) in machine condition monitoring and diagnosis. Chen et al. [12] utilized logistic regression model to process vibration signals for cutting tool monitoring. Karandikar et al. [13] evaluated the performance of two different machine learning methods in predicting tool life curve. Yang et al. [14] established a v-support vector regression (v-SVR) model to study the relationship between fused features and actual tool wear for tool wear monitoring. Zhang et al. [15] used a least square support vector machine (LS-SVM) to predict tool wear of cutting edge position under joint effect of machining conditions. Kong et al. [16] presented a Gaussian process regression technique for accurately monitoring flank wear width. Kong et al. [17] developed a Gaussian mixture hidden Markov models to determine the tool wear states. 
Zhou et al. [18] utilized extension neural networks (ENNs) to fast recognize cutting tool conditions with high precision.

The machinery health prognostic program generally follows a similar technical process: first is extracting artificially designed features from acquired signals for determining state change of tool, and then establishing the nonlinear mapping function between extracted features and tool state by regression methods. But in the above methods, there are two main shortcomings in ANNs-based fault prognosis approaches. First, the inputs rely heavily on signal preprocessing techniques. Second, the simple architecture of ANNs lacks sufficient breadth and depth to map complex nonlinear relationship. The development of deep learning have relieved the above problems to a certain extent $[19,20]$. Deep learning can adaptively learn hierarchical representation without extracting the fault features manually $[21,22]$, which is beneficial to improve adaptability. In addition, more hidden layers are added to process nonlinear inputs, which is more likely to learn deeper hidden information and then to improve prediction accuracy. Deep learning models have attracted increasing attention in tasks of fault diagnosis and prognosis. Jia et al. [2323] designed a deep neural networks (DNNs) based method for fault diagnosis in rolling element bearings and planetary gearboxes. Shao et al. [24] constructed a convolutional deep belief network for fault diagnosis of rolling bearing,which used compressed sensing (CS) for reducing data amount. Wu et al. [25] utilized bidirectional long short-term memory neural network (BiLSTM) to deal with singular value decomposition features to predict current tool wear value. Zhao et al. [26] proposed a deep residual network with dynamically weighted wavelet coefficients for planetary gearbox fault diagnosis.

Different from above-mentioned approaches, deep reinforcement learning can directly map raw extracted features to corresponding tool wear state, which is helpful to further improve intelligence of prediction methods. Combining the advantages of deep learning and reinforcement learning, deep reinforcement learning is able to construct environment according to extracted features, from which artificial agents can learn observations and rewards. Reinforcement learning gives agents the ability to interact with its environment, while deep learning enables agents to learn the better decisions to scale to problems with high-dimensional state and action spaces [27]. As the most significant breakthrough in the field of artificial intelligence, AlphaGo, proves the effectiveness of DRL mechanism [28]. Since then DRL algorithms have been widely applied in domain of modern manufacturing systems, natural language processing, and automated machine learning. In modern manufacturing systems, as a common solution for optimization problems by trial and error, DRL has already been used in fields such as robot training [29,30], management of Industrial Internet of Things [31,32], dynamic scheduling of flexible job shop [33,34], and machinery fault diagnosis [35]. While, how to transfer a DRL network to an effective application against the limited availability of training data for RUL prediction is still a hotspot issue in accurate prediction of tool RUL.

To overcome the deficiencies of limited data and further improve accuracy and intelligence of prediction methods, a deep reinforcement transfer learning (DTRL) method is researched in this paper. This novel approach first extract local features from consecutive time series data to reduce the network size. Then in DRL prediction method, LSTM network is adopted to construct the value function approximation for deeply mining temporal information. A novel strategy of Q-function update and transfer has been proposed to guarantee transferability of trained network to new domain. Finally, tool wear experiments are carried out and the effectiveness of the proposed method is verified by analyzing the datasets.

The rest of this paper is organized as follows. Theoretical foundation about DRL is introduced in Section 2, based on which the framework of proposed DTRL method is shown in Section 3. Tool wear experiments and case study on RUL prediction are conducted in Section 4. Model comparison and validation are shown in Section 5. Finally, the conclusions are summarized in Section 6.

\section{Theoretical foundation}

Deep reinforcement learning (DRL) is a branch of dynamic programming based reinforcement learning, in which agents interact with the environment while learning. The interactive learning process can be modeled by Markov decision process (MDP) expressed by a tuple:

$M=(S, A, T, R, \gamma)$

where $S$ is a finite set of states, $A$ is a finite set of actions, $T: S \times A \times S \rightarrow[0,1]$ is the transition function, $R: S \times A \times S \rightarrow \mathrm{R}$ is the reward function, and $\gamma \in[0,1)$ is the discount factor. $\pi: S \times A \rightarrow[0,1]$ is a deterministic policy to demonstrate the probability of the action. The state-action value function $Q: S \times A \rightarrow \mathrm{R}$ following policy $\pi$ can be defined as:

$Q^{\pi}(s, a)=E_{\pi}\left[\sum_{t=0}^{\infty} \gamma^{t} r_{t+k} \mid s_{k}=s, a_{k}=a\right]$

The goal of each MDP is to find an optimal policy $\pi^{*}$, and it owns expected return $V^{*}(s)$ and value function $Q^{*}(s, a)$. The Q-function in DRL satisfies the Bellman optimality equation. Therefore, an optimal state-action value satisfies the following equation:

$Q^{*}(s, a)=\sum_{s^{\prime} \in S} T\left(s, a, s^{\prime}\right)\left(R\left(s, a, s^{\prime}\right)+\gamma V^{*}\left(s^{\prime}\right)\right)$

One of the most popular methods to estimate the value of state-action is the Q-learning algorithm. The basic idea of deep Q-learning is to estimate Q-values based on rewards 
and the agent's Q-value function. The Q-update rule for model-free online learning can be expressed as:

$$
Q^{\prime}(s, a)=Q(s, a)+\alpha\left[R+\gamma \max _{a^{\prime}} Q\left(s^{\prime}, a^{\prime}\right)-Q(s, a)\right\rfloor
$$

where $\alpha$ is the learning rate. The max error is utilized to evaluate the quality of Q-function:

$$
\text { error }=\left(R+\gamma\left|\max _{a^{\prime}} Q(s, a)-Q^{*}(s, a)\right|\right)
$$

\section{Proposed DTRL architecture}

In the practical application of deep learning, there are two common problems: the first is to deal with extremely large state space of tabular Q-learning in time series analysis, the second is to process unlabeled data. To solve the above issues, the DTRL architecture is designed, which combines deep learning with transfer reinforcement learning. More specifically, the DTRL method inputs the current state and action, then adopts a LSTM to estimate the value of $Q(s, a)$, and at last transfer the Q-values to another LSTM network. The estimated value of $Q(s, a)$ is defined as

$$
Q(s, a)=\mathrm{E}\left[R+\gamma \max _{a^{\prime}} Q\left(s^{\prime}, a^{\prime}\right)\right]
$$

\subsection{Parameter reinforcement Q learning}

To solve the problem caused by large state space in the RUL prediction and improve the generalization ability of deep Qfunction, Deep Q-Network (DQN) is adopted. DQN is a model parameterized by weights and biases collectively denoted as $\theta$. In DQN, Q-values at each training iteration $t$ can be denoted by $Q_{\theta_{t}}(s, a)$. More specifically, Q-values are estimated by performing forward propagation then querying the output nodes. To obtain the estimation of Q-values shown in (6), the proposed DTRL method adopts the experience replay method [36]. After one prediction, the experiences at current time step, denoted as $e_{t}=\left(s, a, R, s^{\prime}\right)$, are recorded in the replay memory $M=\left\{e_{1}, e_{2}, \ldots, e_{t}\right\}$, and then sampled randomly at training time. Instead of updating Q-table lookups, now the network parameters $\theta$ are updated with stochastic gradient algorithm to minimize the differentiable loss function:

$$
L\left(\theta_{t}\right)=\mathrm{E}_{\left(s, a, r, s^{\prime}\right)}\left[\left(\begin{array}{c}
\left.\left.R+\gamma \max Q_{a_{t}^{\prime}}\left(s^{\prime}, a^{\prime}\right)-Q_{\theta_{t}}(s, a)\right)^{2}\right] \\
a^{\prime}
\end{array}\right.\right.
$$

When the Q-function changes quite rapidly, the updates may oscillate or diverge. At the same time, when there are too many iterations, the algorithms will be inefficient. To avoid the above problems, the proposed DTRL method adopt the fixed Q-targets method. Instead of using the latest para- meters $\theta_{t}$ to calculate the maximum possible reward of the next state $\gamma \max _{a^{\prime}} Q_{\theta_{t}}\left(s^{\prime}, a^{\prime}\right)$, we update the parameters $\theta^{\prime}$ every certain iterations. Differentiating the loss function with respect to the parameters, the gradient is shown as follows:

$$
\nabla_{\theta_{t}} L\left(\theta_{t}\right)=\mathrm{E}_{\left(s, a, r, s^{\prime}\right)}\left[\left(y_{\theta_{t}}-Q_{\theta_{t}}(s, a)\right) \nabla_{\theta_{t}} Q_{\theta_{t}}(s, a)\right]
$$

where $y_{\theta_{t}}=R+\gamma \max Q_{a^{\prime}}\left(s^{\prime}, a^{\prime}\right)$ is the stale update target.

\subsection{Parameter reinforcement $Q$ learning}

In the DQN-based RUL prediction method, we use densely connected networks to capture the state correlations. However, the real-word RUL prediction tasks also exist temporal correlations and vanishing gradient problems, which may result in degradation of DQN's performance. Therefore, in the proposed DTRL method, LSTM layers are adopted instead of dense layers to carry information across many timesteps. More specifically, the Q-funciton in the DTRLbased RUL prediction can be defined as

$Q_{\theta_{t}}\left(s, a, h_{t-1}\right)$

where $h_{t-1}$ is the supplementary input calculated by LSTM layers according to the previous information. Consequently, the gradient of the loss function is shown as

$$
\begin{aligned}
& \nabla_{\theta_{t}} L\left(\theta_{t}\right)= \\
& \mathrm{E}_{\left(s, a, r, s^{\prime}\right)}\left[\left(y_{\theta_{t-1}}-Q_{\theta_{t}}\left(s, a, h_{t-1}\right)\right) \nabla_{\theta_{t}} Q_{\theta_{t}}\left(s, a, h_{t-1}\right)\right] \\
& y_{\theta_{t-1}}=R+\gamma \underset{a^{\prime}}{\max Q_{\theta_{t}}\left(s^{\prime}, a^{\prime}, h^{\prime}{ }_{t-1}\right)}
\end{aligned}
$$

The DTRL method adopts LSTM layers instead of simple dense layers to process temporal series. As shown in Fig. 1, LSTM layers take as input temporal sequential experiences $e=\left\{e_{1}, e_{2}, \ldots, e_{t}\right\}$ and Q-values are calculated after the output layer. In the practical prediction process, as LSTM layers save multi-timesteps information, we are supposed to choose experiences traces with certain length instead of single experience.

\subsection{Deep Q transfer learning}

After deep reinforcement Q learning, we can calculate the Qfunction of each state action pair, which can help to select the values with the least error in the prediction tasks. But the above algorithm requires a large amount of experimental fault datasets, which is almost impossible to be acquired at the beginning of practical manufacturing. Hence the transfer learning method is introduced to reduce the amount of training datasets and make full use of the trained Q-function. In the RUL prediction tasks, the proposed DTRL method transfers the Q-function calculated from different tool tasks 
Fig. 1 Architecture of the deep transfer reinforcement learning network

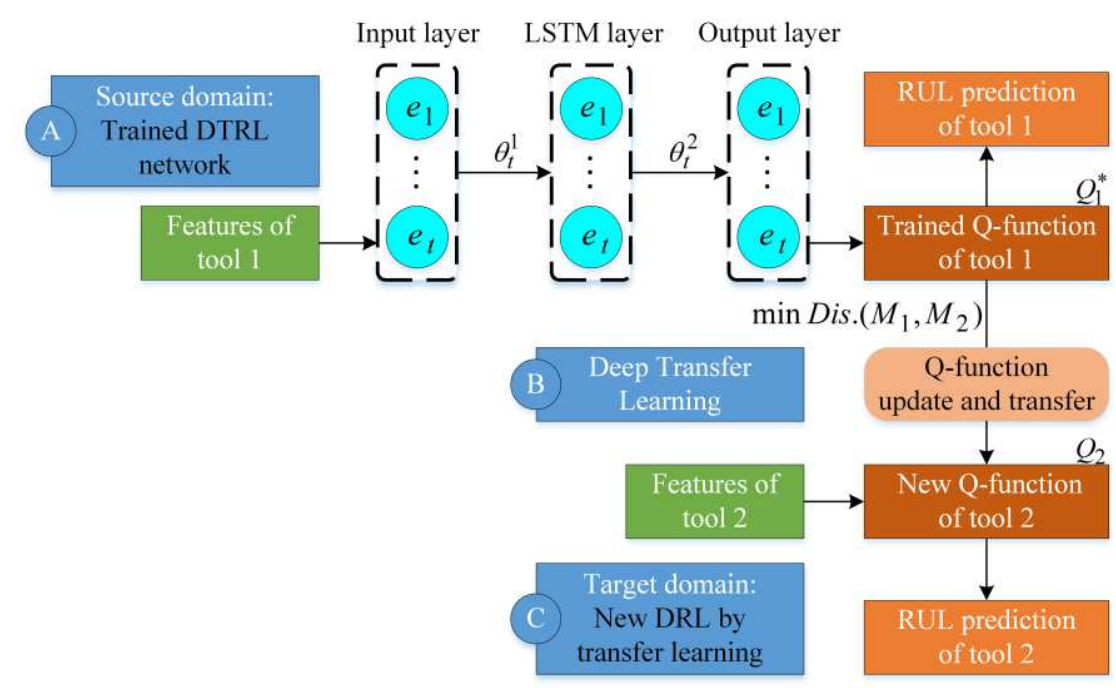

(source domain) to another tool task (target domain), which aims to improve the learning ability in new prediction tasks by introducing knowledge from a similar learned prediction task.

According to the DRL, the tool RUL prediction tasks can be defined as $M=(S, A, T, R, \gamma)$, and the tasks are different in transition function $T$, reward function $R$ and discount factor $\gamma$. As shown in Fig. 1. The source domain, denoted as $M_{1}=\left(S, A, T_{1}, R_{1}, \gamma_{1}\right)$, is the trained DRL network, and the target domain, denoted as $M_{2}=\left(S, A, T_{2}, R_{2}, \gamma_{2}\right)$, is the new DRL network. Assume $Q_{1}{ }^{*}$ and $Q_{2}{ }^{*}$ are corresponding optimal Q-functions. The main goal of the DTRL method is using the information of $M_{1}$ and $Q_{1} *$ to update $Q_{2}$ and improve training speed of $M_{2}$ while ensuring the prediction accuracy.

To learn similar and joint Q-function for the source domain and the target domain, the distance between Qfunctions calculated by two networks are minimized. The distance between two tasks is defined as

$$
\begin{aligned}
& \text { Dis. }\left(M_{1}, M_{2}\right) \\
& =\max _{s, a}\left|Q_{1}^{*}(s, a)-Q_{2}^{*}(s, a)\right| \\
& =\left\|Q_{1}^{*}-Q_{3}^{*}+Q_{3}^{*}-Q_{4}^{*}+Q_{4}^{*}-Q_{2}^{*}\right\|_{\infty} \\
& \leq \frac{\left\|R_{1}-R_{2}\right\|_{\infty}}{1-\gamma_{1}}+\frac{\gamma_{2}\left\|R_{2}\right\|_{\infty}}{\left(1-\gamma_{1}\right)^{2}}\left\|T_{1}-T_{2}\right\|_{\infty}+\frac{\left|\gamma_{1}-\gamma_{2}\right|}{\left(1-\gamma_{1}\right)\left(1-\gamma_{2}\right)}\left\|R_{2}\right\|_{\infty}
\end{aligned}
$$

By minimizing the distance between two tasks, Qfunctions learned in the target domain are restricted to be similar to that of the source domain, consequently deep Q transfer learning is achieved. According to the distance between two tasks calculated in Eq. (12), the Q-function update in the new DRL network is performed. Finally, the forward iteration process is implemented in the new DRL network, and the RUL prediction results of the target domain are output.

The DTRL network is realized through above mentioned steps, and the whole architecture is shown in Fig. 1.

\subsection{DTRL for RUL prediction}

In the real prediction tasks, the first step is feature extraction and data preprocess, then take the results as the input of the DTRL method. We model the mapping function between the extracted features and the corresponding RUL sampling points, which can be expressed as

$$
\left[\begin{array}{rrrr}
f_{11} & f_{12} & \Lambda & f_{1 m} \\
f_{21} & f_{22} & \mathrm{~K} & f_{2 m} \\
\mathrm{M} & \mathrm{M} & \mathrm{O} & \mathrm{M} \\
f_{n 1} & f_{n 2} & \mathrm{~K} & f_{n m}
\end{array}\right] \Rightarrow\left[\begin{array}{c}
t_{1} \\
t_{2} \\
\mathrm{M} \\
t_{n}
\end{array}\right]
$$

where $\left[f_{1}, f_{2}, \ldots, f_{m}\right]$ denote the extracted features with $m$ denoting numbers of input features at a certain point in time, and $\left[t_{1}, t_{2}, \ldots, t_{n}\right]^{T}$ denote the RUL with $n$ denoting sampling time points during run-to-failure. In the real tool processing, the tool performance is generally nonlinear due to the influence of nonlinear factors such as crack growth in material and sudden change of machining parameters. Therefore, a nonlinear activation function $\varphi(*)$ is adopted to fit mapping function between feature matrix and RUL series.

$$
R U L=\varphi(F) \omega+b
$$

where $R U L=\left[t_{1}, t_{2}, \ldots, t_{n}\right]^{T}$ is time sample series, $\varphi(F)$ is a nonlinear feature matrix, $\omega$ is the corresponding weights for nonlinear regression and $b$ is the bias.

The DTRL-based RUL prediction algorithm is summarized in Algorithm 1. 


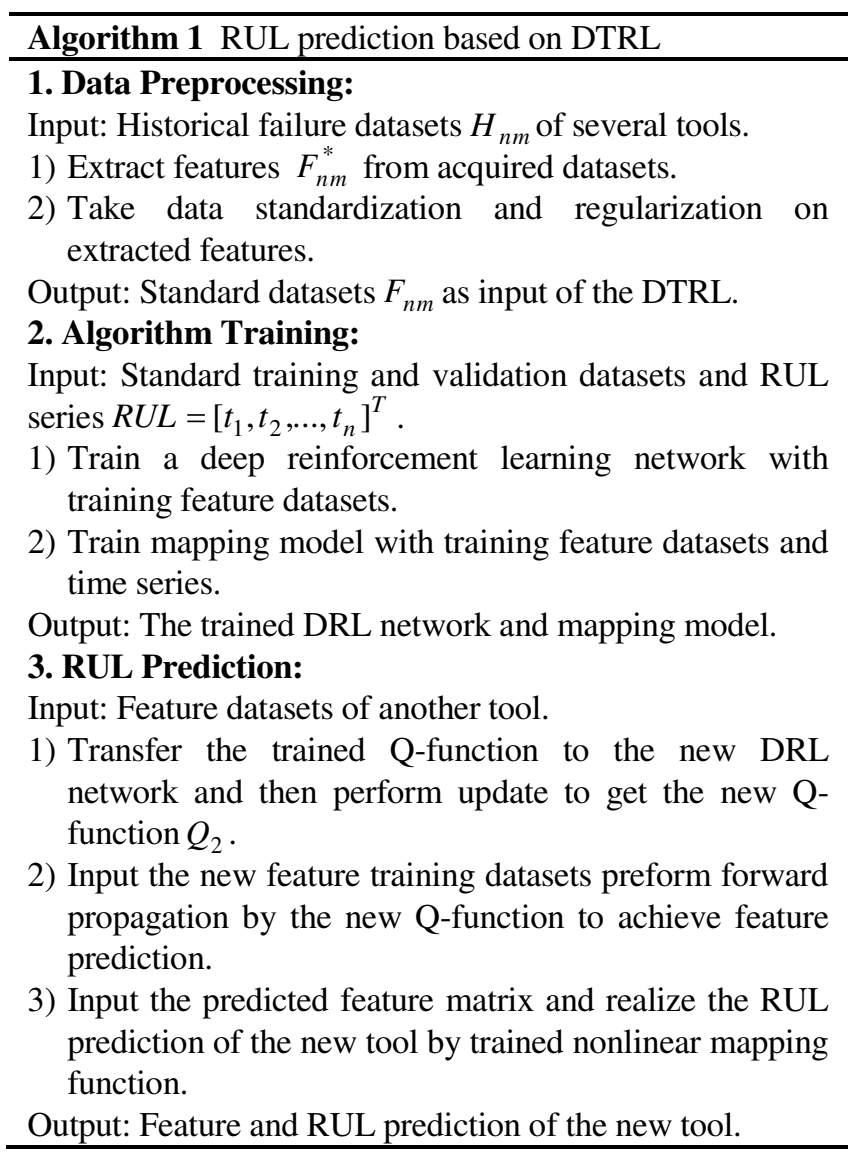

\section{Experiment validation}

\subsection{Benchmarking data description}

To validate effectiveness of the proposed DTRL method, extensive experiments on tool performance degradation are conducted. The experiments are carried out on a high-speed CNC machine, as shown in Fig. 2. The cutting tools are endmilling cutters with TiAIN coating and the material of cutting workpiece is CR12moV. The key signals collected synchronously are cutting force and vibration signals. Other experimental conditions for tool processing are as follows: The spindle rotation frequency was $3000 \mathrm{r} / \mathrm{min}$; the cutting depth in radial and axial direction was $0.5 \mathrm{~mm}$; the sampling frequency was $10 \mathrm{kHz}$ and a run-to-failure tool produced a total of 312 cutting segments. A Kistler compact multicomponent dynamometer was mounted to collect cutting force signals in real time, and a DAQ Elsys TraNET 404S8 was used to collect vibration signals synchronously. Therefore, six kinds of sensor signals were acquired, including force in three directions and vibration in three directions.

\subsection{Data preprocessing and DRL training}

In order to make the raw sensor signal more amenable to models, the first step is data preprocessing, including feature extraction and normalization. The simplest way to prevent overfitting is to reduce the memorization capacity of the model. Therefore, orthogonal wavelet packet transform (OWPT) is used to extract the features of cutting force and vibration signals, so as to reduce the input data without losing information. In this paper, to acquire discriminated feature, six-level OWPT using db1 is adopted to calculate the energy of each sub-band. Each feature can be separated into 64 sub-bands, of which the energy is defined as

$$
E_{i}=\sum_{m=1}^{n}\left|x_{i, m}\right|
$$

where $x_{i, m}$ is wavelet coefficient in scale $2^{i}$, and $n$ is the oscillation parameter. The number of energy can effectively reflect the tool wear state, and the energy spectrum of cutting force and vibration signal in each cutting segment can be converted into the form shown in Fig. 3. Then, each energy feature is normalized independently to accelerate model convergence.

After data preprocessing, frequency spectrum of the signals monitored from tool wear process is extracted as weighted average energy, which is used as input to DTRL for model training and RUL prediction. To configure network
Fig. 2 CNC machine monitoring systems for tool wear data acquisition.

(a) CNC machine tool.

(b) Tool wear monitoring system.

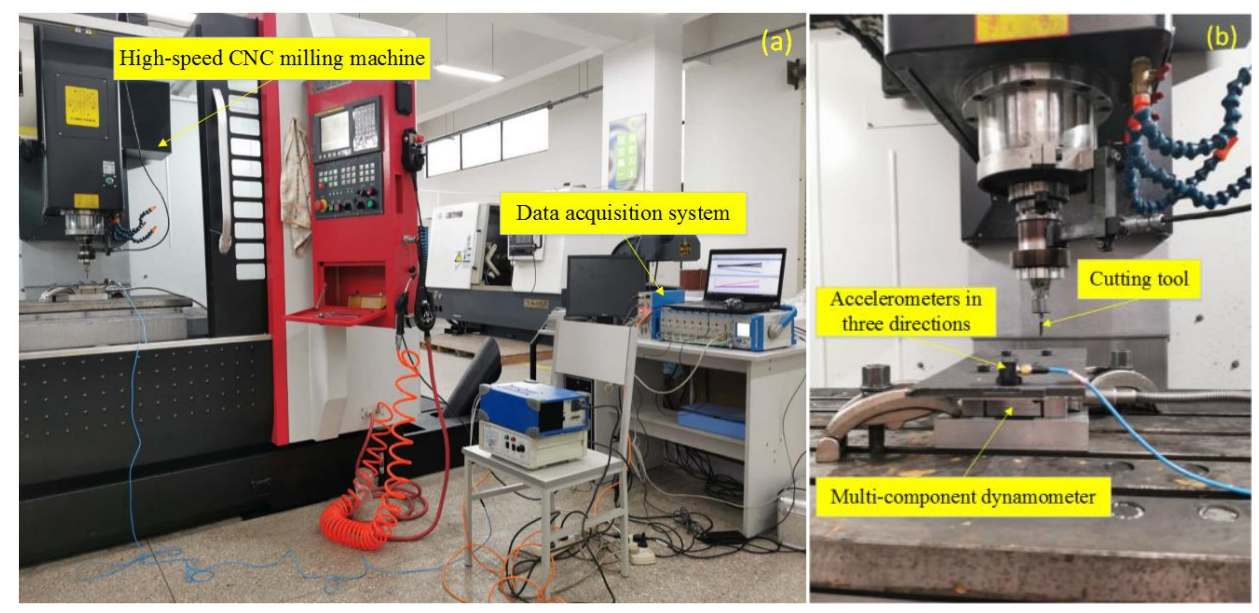



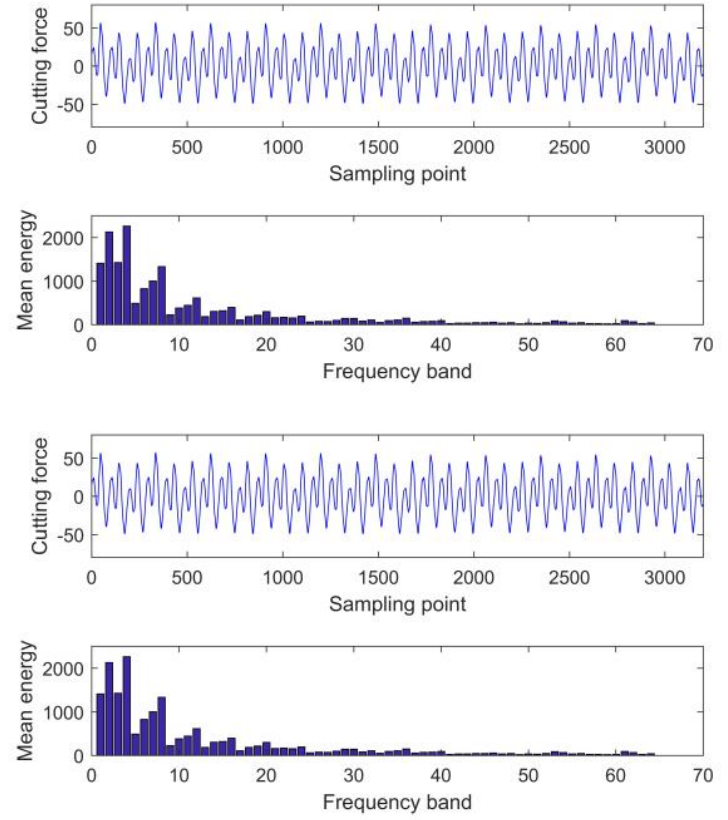

Fig. 3 Cutting force signals, vibration signals and their energy the wavelet packet

structure of the first Q-function of tool1, the number of nodes in both input and output layers is set to be 6 , which is equal to the number of extracted features. The discount factor $\gamma$ is set to 0.9 and the learning rate $\alpha$ is set to 0.05 in Eq.(10). In order to ensure that the training results converge to the global optimum, the learning rate is set to decrease exponentially with the number of training epochs. Then to accelerate the convergence speed of network in training process, RMSprop is used to optimize gradient descent process. Finally, training the Q-function on the training dataset by conducting updating until the Q-function is converged.

\subsection{Tool Prediction Results}

After model training stage, a well trained Q-function is obtained and applied in next testing stage. The network structure of the trained Q-function is first updated and transferred to establish a new DRL network for feature learning of tool2. Then the sensor data of tool2 is preprocessed and the extracted energy features are input to the new DRL network. After a forward iteration process, for clarity, the force and vibration energy features learned by the DTRL network is independently shown in Fig. 4. The $\mathrm{X}$ axis represents the cutting steps of tool2, and the $\mathrm{Y}$ axis represents the corresponding energy features. It can be seen that with increase in time of tool running, the predicted value has a similar increasing trend with the real value. Increasing trend of each extracted feature is consistent with that of tool wear degree in tool2, which shows the extracted features can effectively reflect the degradation of tool performance. The
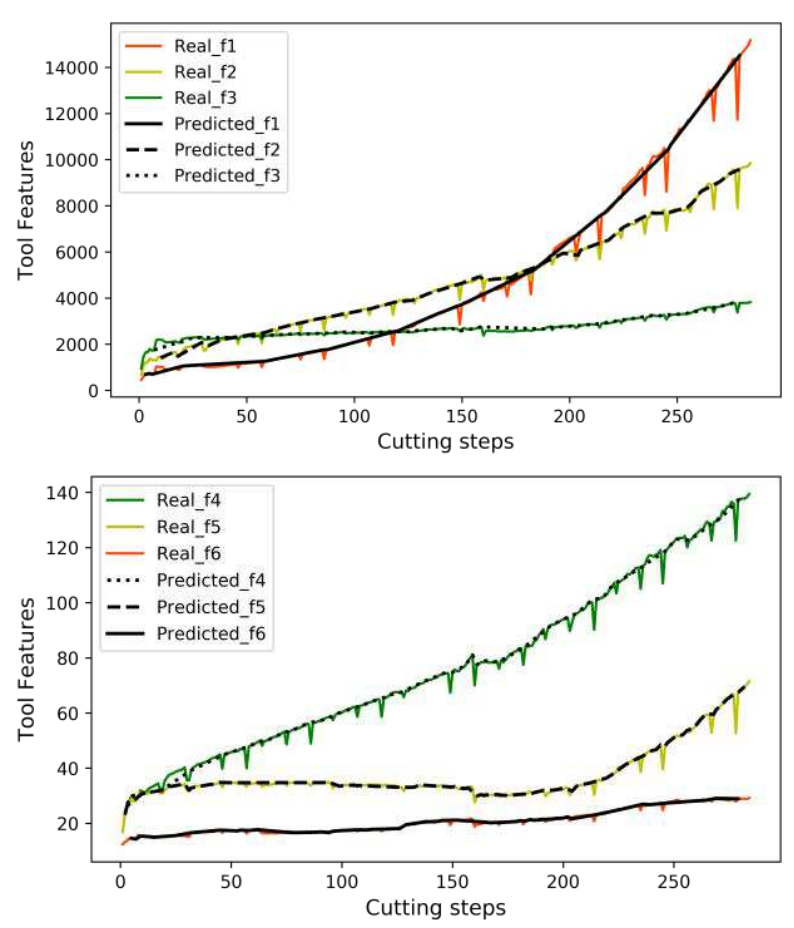

Fig. 4 Predicted features of tool2 learned by the DTRL network

result demonstrates that DTRL network is an effective feature prediction model for tool wear monitoring.

To further perform RUL prediction by the DTRL network for tool2, first is establishing nonlinear mapping function between predicted energy features and time series of RUL. Support vector regression (SVR) is used to construct the regression model, in which the kernel function choosing radial basis function (RBF). The kernel function can be expressed as

$$
K\left(x, x_{i}\right)=\exp \left(-\gamma\left\|x-x_{i}\right\|^{2}\right)
$$

where $\gamma$ denoting the width parameter. The energy features and corresponding RUL time of tooll form the training samples. In order to minimize the training error, the width parameter $\gamma$ is adjusted to 30 .

Finally, the energy features of tool 2 are input to the trained regression model to further evaluate the effectiveness of the proposed DTRL method. RUL prediction result is shown in Fig. 5, and it can be seen that the predicted curve is very close to the real curve. To quantitatively measure the performance of DTRL method on RUL prediction tasks, two indicators for evaluating prediction precision are utilized including mean absolute error (MAE) and root mean squared error (RMSE). The corresponding equations to calculate errors are expressed as follows:

$$
M A E=\frac{1}{N} \sum_{i=1}^{N}\left|\tilde{T}_{i}-T_{i}\right|
$$




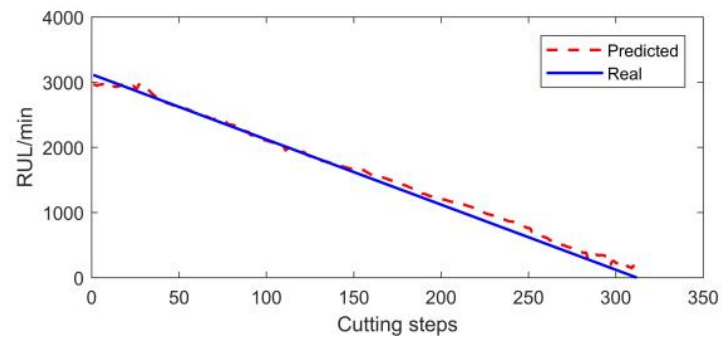

Fig. 5 RUL Prediction result for tool2

Table 1 MAE and RMSE achieved by DTRL for tool2

\begin{tabular}{cc}
\hline MAE & RMSE \\
\hline 16.54 & 20.23 \\
\hline
\end{tabular}

$\operatorname{RMS} E=\sqrt{\frac{1}{N} \sum_{i=1}^{N}\left(\tilde{T}_{i}-T_{i}\right)^{2}}$

where $\tilde{T}_{i}$ and $T_{i}$ are true and predicted tool RUL, respectively.

The prediction error is shown in Table 1. It can be seen that the validation MAE and RMSE translate to RUL of $16.54 \mathrm{~min}$ and $20.23 \mathrm{~min}$ after denormalization. The results show a low prediction error is acquired and demonstrate the effectiveness of DTRL method on RUL prediction tasks.

\section{Model Comparison and Validation}

In Section 4, the proposed DTRL method is used to predict RUL of a new tool. The tool types and cutting conditions are rather limited. To verify effectiveness of deep $Q$ transfer learning (DQTL) strategy in improving prediction performance, a comparison research without transfer learning for tool2 is performed. Furthermore, to investigate the generalization ability of the proposed DTRL method, more experiments are conducted.

\subsection{Model Comparison}

The energy features of tool2 are input to the trained regression model without DQTL. RUL prediction result is shown in Fig. 6, which shows a larger prediction error compared with Fig. 5, especially from middle stage of cutting processing. The corresponding prediction error is shown in Table 2. It can be seen that the quantitative error is higher compared with that in Table 1 , which demonstrates the proposed DQTL as an effective method to improve prediction accuracy of DRL network.

\subsection{Model Validation}

To verify generality of the method, two kinds of experiments are extended. One kind is utilizing a new type of tool under

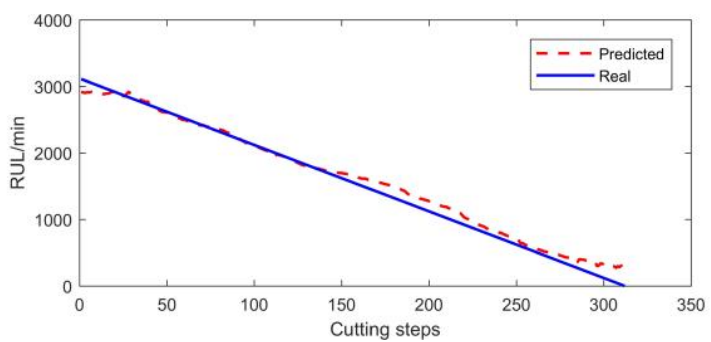

Fig. 6 RUL Prediction result for tool 2 without deep Q transfer learning

Table 2 MAE and RMSE for tool2 without deep Q transfer learning

\begin{tabular}{cc}
\hline MAE & RMSE \\
\hline 20.61 & 26.29 \\
\hline
\end{tabular}

Table 3 MAE and RMSE of entend experiments

\begin{tabular}{lcc}
\hline & MAE & RMSE \\
\hline Tool with different diameter & 26.12 & 33.68 \\
\hline Tool under different cutting conditions & 23.82 & 30.43 \\
\hline
\end{tabular}

same cutting conditions, in which the diameter of original tool is $6 \mathrm{~mm}$ and the diameter of new type is $8 \mathrm{~mm}$. The other kind is under different cutting conditions, in which the spindle rotation frequency is $10000 \mathrm{r} / \mathrm{min}$ and the cutting depth in radial and axial direction was $0.25 \mathrm{~mm}$.

Comparing parameters include tool geometries and cutting parameters. During the experiments, tool geometries are fixed, thus cutting parameters have greater influence on force and vibration amplitude. Cutting parameters, including spindle speed and cutting depth, may cause different dynamic ranges under similar cutting conditions. To eliminate the above effects and improve the generalization ability of the proposed model, a zero-centered step is first applied to the collected signals. Then, wavelet packet energy features are extracted from the cutting force and vibration signals of each sampling point. In this paper, 6-level wavelet packet decomposition is adopted, thus the harmonics are decomposed into 64 wavelet packet energy coefficients. Finally, energy coefficient with highest weight is input into the model instead of original signal (see Fig. 3), so the model is robust to various cutting conditions.

Table 3 shows the MAE and RMSE translated to RUL in two extended experiments. The results indicate that the DTRL method can effectively predict tool wear under various cutting conditions.

\section{Conclusion}

In this paper, a DTRL method is introduced to conduct deep Q-function transfer in reinforcement learning network. Two strategies, including introducing LSTM into DQN, Qfunction update and transfer, is designed to realize DTRL network. Experimental results demonstrate the effectiveness 
of DTRL network for tool wear monitoring and RUL prediction. Three conclusions are summarized as following:

(1) Compared with traditional reinforcement learning methods, accuracy of RUL prediction can be improved by deep Q-function transfer learning.

(2) The energy features decomposed by WPD from original signals can make DTRL network robust to different cutting conditions.

(3) The proposed DTRL is trained by a set of historical failure data and transferred to various tool diameter and cutting conditions. Therefore, DTRL is able to promote successful applications of RUL prediction of new tools with limited historical failure data.

\section{References}

1. Liao X, Zhou G, Zhang Z, Lu J, Ma J (2019) Tool wear state recognition based on GWO-SVM with feature selection of genetic algorithm. Int J Adv Manuf Technol 104(1-4):1051-1063

2. Aghazadeh F, Tahan A, Thomas M (2018) Tool condition monitoring using spectral subtraction and convolutional neural networks in milling process. Int J Adv Manuf Technol 98(9-12):3217-3227

3. Wang G, Qian L, Guo Z (2012) Continuous tool wear prediction based on Gaussian mixture regression model. Int J Adv Manuf Technol 66(912):1921-1929

4. Si X, Wang W; Hu C (2013) A Wiener-process-based degradation model with a recursive filter algorithm for remaining useful life estimation. Mech Syst Signal Process 35(1-2):219-237

5. Yan H, Zhou J, Pang C (2015) Gamma process with recursive MLE for wear PDF prediction in precognitive maintenance under aperiodic monitoring. Mechatronics 31:68-77

6. Wang J, Wang P, Gao R (2015) Enhanced particle filter for tool wear prediction. J Manuf Syst 36: 35-45

7. Li C, Sanchez R, Zurita G (2016) Gearbox fault diagnosis based on deep random forest fusion of acoustic and vibratory signals. Mech Syst Signal Process 76-77: 283-293.

8. Javed K, Gouriveau R, Li X, Zerhouni N (2016) Tool wear monitoring and prognostics challenges: a comparison of connectionist methods toward an adaptive ensemble model. J Intell Manuf 29(8): 1873-1890

9. Dou J, Xu C, Jiao S, Li B, Zhang J, Xu X (2019) An unsupervised online monitoring method for tool wear using a sparse auto-encoder. Int J Adv Manuf Technol 106:2493-2507

10. Sun C, Wang P, Yan R (2019) Machine health monitoring based on locally linear embedding with kernel sparse representation for neighborhood optimization. Mech Syst Signal Process 114:25-34

11. Widodo A, Yang B (2007) Support vector machine in machine condition monitoring and fault diagnosis. Mech Syst Signal Process 21(6):2560-2574.

12. Chen B, Chen X, Li B (2011) Reliability estimation for cutting tools based on logistic regression model using vibration signals. Mech Syst Signal Process 25(7):2526-2537.

13. Karandikar J (2019) Machine learning classification for tool life modeling using production shop-floor tool wear data. Procedia Manuf 34:446-454

14. Kong D, Chen Y, Li N, Tan S (2016) Tool wear monitoring based on kernel principal component analysis and v-support vector regression. Int J Adv Manuf Technol 89(1-4):175-190
15. Zhang C, Zhang H (2016) Modelling and prediction of tool wear using LS-SVM in milling operation. Int J Comput Integ Manuf 29(1):76-91

16. Kong D, Chen Y, Li N (2018) Gaussian process regression for tool wear prediction. Mech Syst Signal Process 104:556-574

17. Kong D, Chen Y, Li N (2017) Force-based tool wear estimation for milling process using Gaussian mixture hiddenMarkov models. Int $\mathrm{J}$ Adv Manuf Technol 92(5-8):2853-2865

18. Zhou Y, Wang T (2018) ENN-based recognition method for tool cutting state. J Comput Sci 27:418-427

19. Serin G, Sener B, Ozbayoglu A, Unver H (2020) Review of tool condition monitoring in machining and opportunities for deep learning. Int J Adv Manuf Technol, 109:953-974

20. Tao Z, An Q, Liu G, Chen M (2019) A novel method for tool conditionmonitoring based on long short-term memory and hidden Markov model hybrid framework in high-speed milling Ti-6Al-4V. Int J Adv Manuf Technol 105(7-8):3165-3182

21. Wang J, Ma Y, Zhang L, Gao R,Wu D (2018) Deep learning for smart manufacturing: Methods and applications. J Manuf Syst 48:144-156

22. Xu X, Wang J, Ming W (2020) In-process tap tool wear monitoring and prediction using a novel model based on deep learning. Int J Adv Manuf Technol 112(1-2):453-466

23. Jia F, Lei Y, Lin J, Zhou X, Lu N (2016) Deep neural networks: A promising tool for fault characteristic mining and intelligent diagnosis of rotating machinery with massive data. Mech Syst Sig Process 72 73:303-315

24. Shao H, Jiang H, Zhang H, Duan W, Liang T, Wu S (2018) Rolling bearing fault feature learning using improved convolutional deep belief network with compressed sensing. Mech Syst Sig Process 100:743-765

25. Wu X, Li J, Jin Y, Zheng S (2020) Modeling and analysis of tool wear prediction based on SVD and BiLSTM. Int J Adv Manuf Technol 106(9-10):4391-4399

26. Zhao M, Kang M, Tang B, Pecht M (2018) Deep residual networks with dynamically weighted wavelet coefficients for fault diagnosis of planetary gearboxes. IEEE Trans Ind Electron 65:4290-4300

27. Arulkumaran K, Deisenroth M, Brundage M, Bharath A (2017) Deep Reinforcement Learning: A Brief Survey. IEEE Signal Process Mag 34(6):26-38

28. Silver D, Huang A, Maddison C (2016) Mastering the game of Go with deep neural networks and tree search. Nature 529:484-489

29. Wang Y, Fang Y, Lou P, Yan J, Liu N (2020) Deep Reinforcement Learning based Path Planning for Mobile Robot in Unknown Environment. J Phys Conf Ser 1576

30. Liu Q, Liu Z, Xu W, Tang Q (2019) Human-robot collaboration in disassembly for sustainable manufacturing. Int J Prod Res 57:40274044

31. Yang H, Zhong W, Chen C (2020) Deep reinforcement learning based energy efficient resource management for social and cognitive internet of things. IEEE Internet Things J 7:5677-5689.

32. Min M, Xiao L, Chen Y (2019) Learning based computation offloading for IoT devices with energy harvesting. IEEE Trans Veh Technol 68:1930-1941

33. Luo S (2020) Dynamic scheduling for flexible job shop with new job insertions by deep reinforcement learning. Appl Soft Comput 91

34. Hu L, Liu Z, Hu W (2020) Petri-net-based dynamic scheduling of flexible manufacturing system via deep reinforcement learning with graph convolutional network. J Manuf Syst 55:1-14.

35. Ding Y, Ma L, Ma J (2019) Intelligent fault diagnosis for rotating machinery using deep Q-network based health state classification: A deep reinforcement learning approach. Adv Eng Inf 42

36. Mnih V, Kavukcuoglu K, Silver D (2015) Human-level control through deep reinforcement learning. Nature 518:529-533. 


\section{Figures}

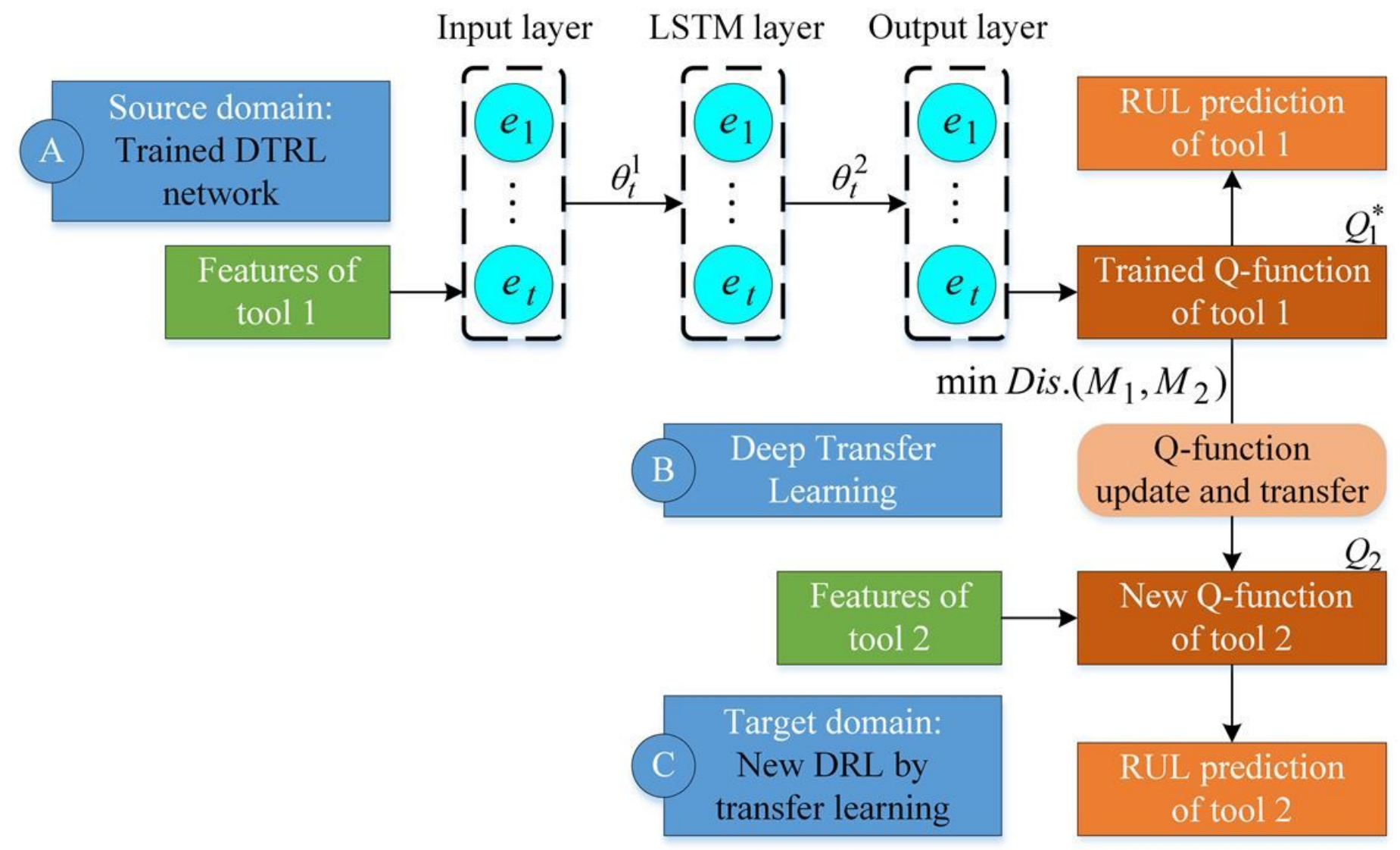

Figure 1

Architecture of the deep transfer reinforcement learning network 


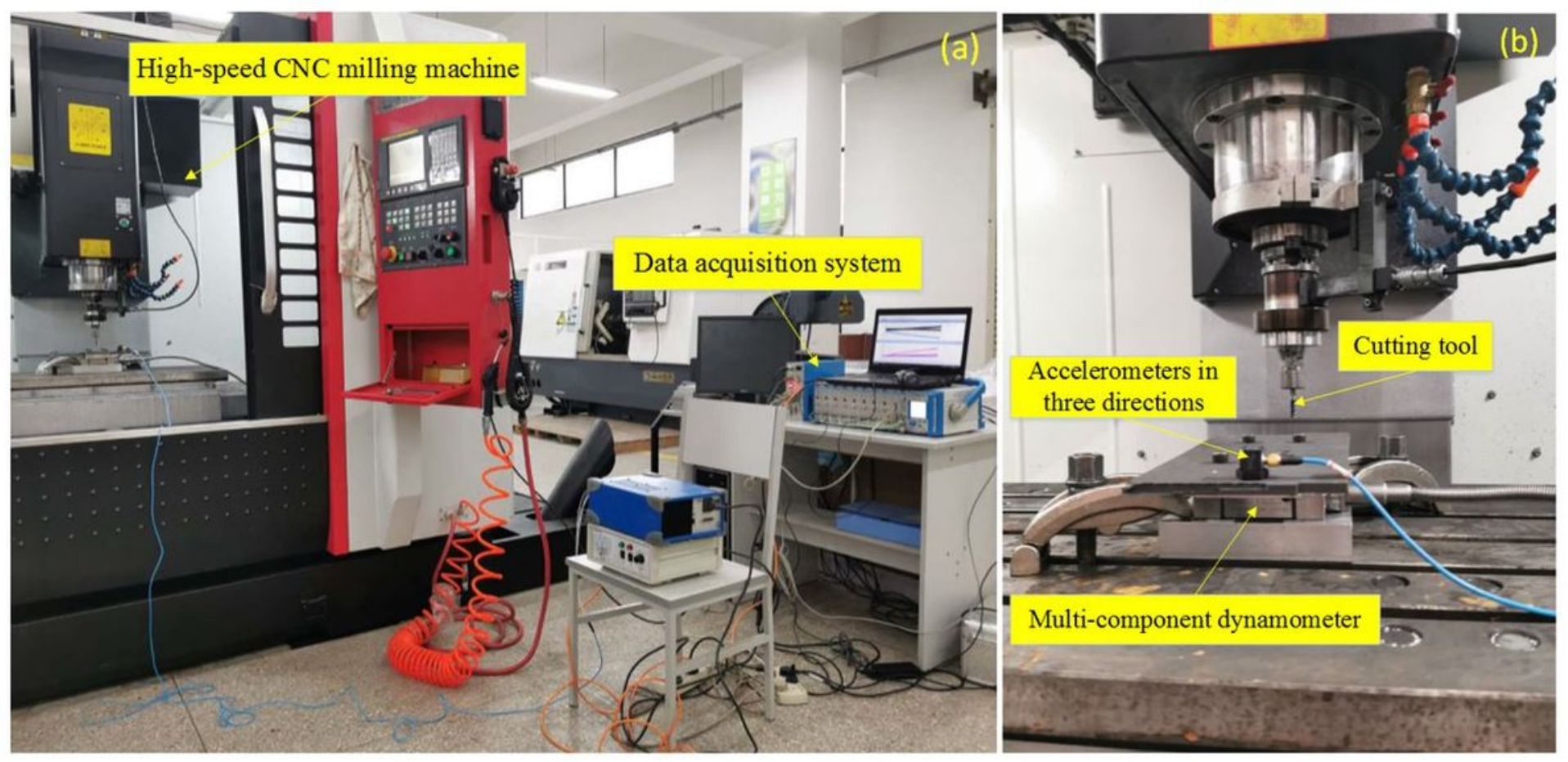

Figure 2

CNC machine monitoring systems for tool wear data acquisition. (a) CNC machine tool. (b) Tool wear monitoring system. 

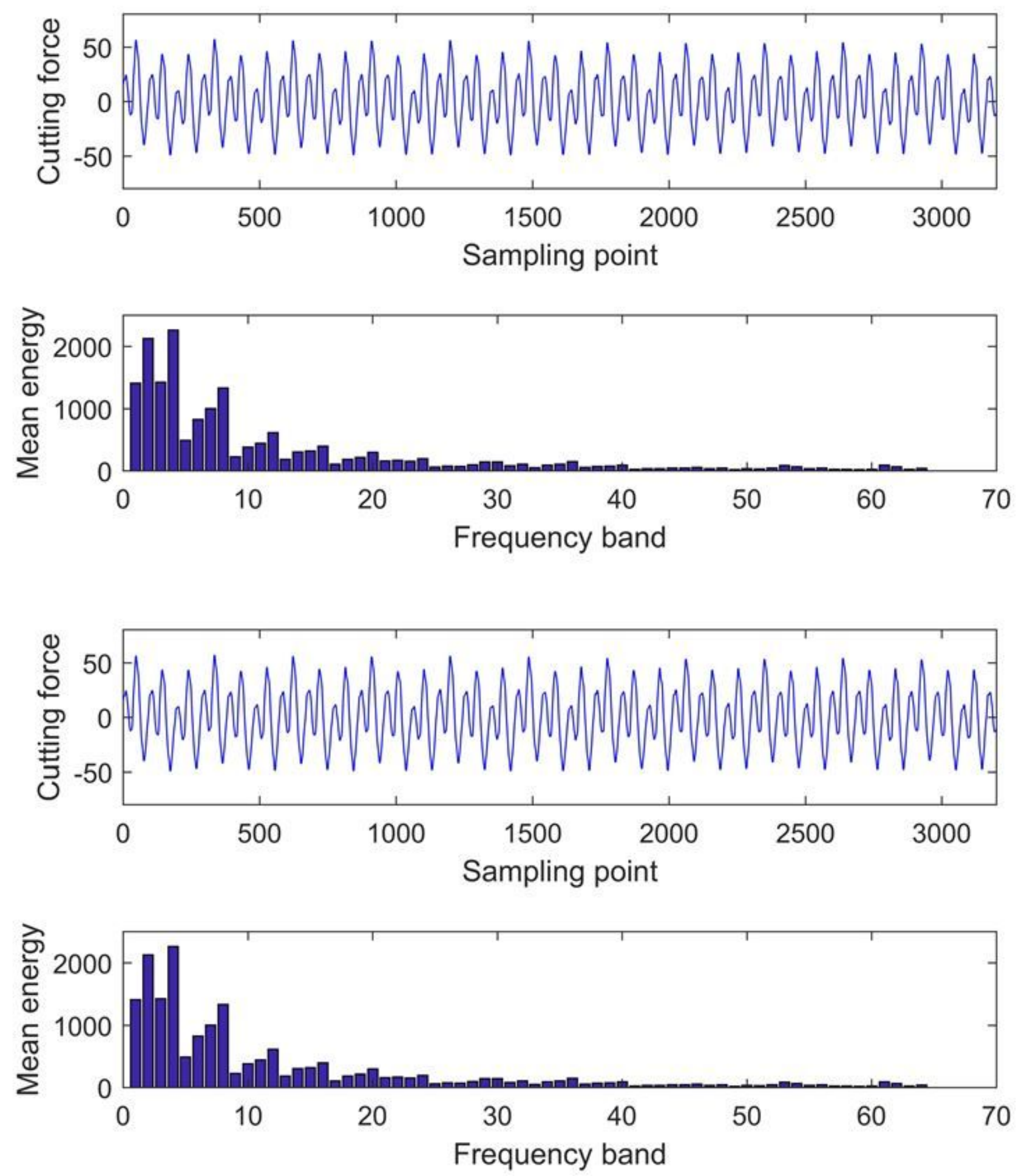

Figure 3

Cutting force signals, vibration signals and their energy the wavelet packet 

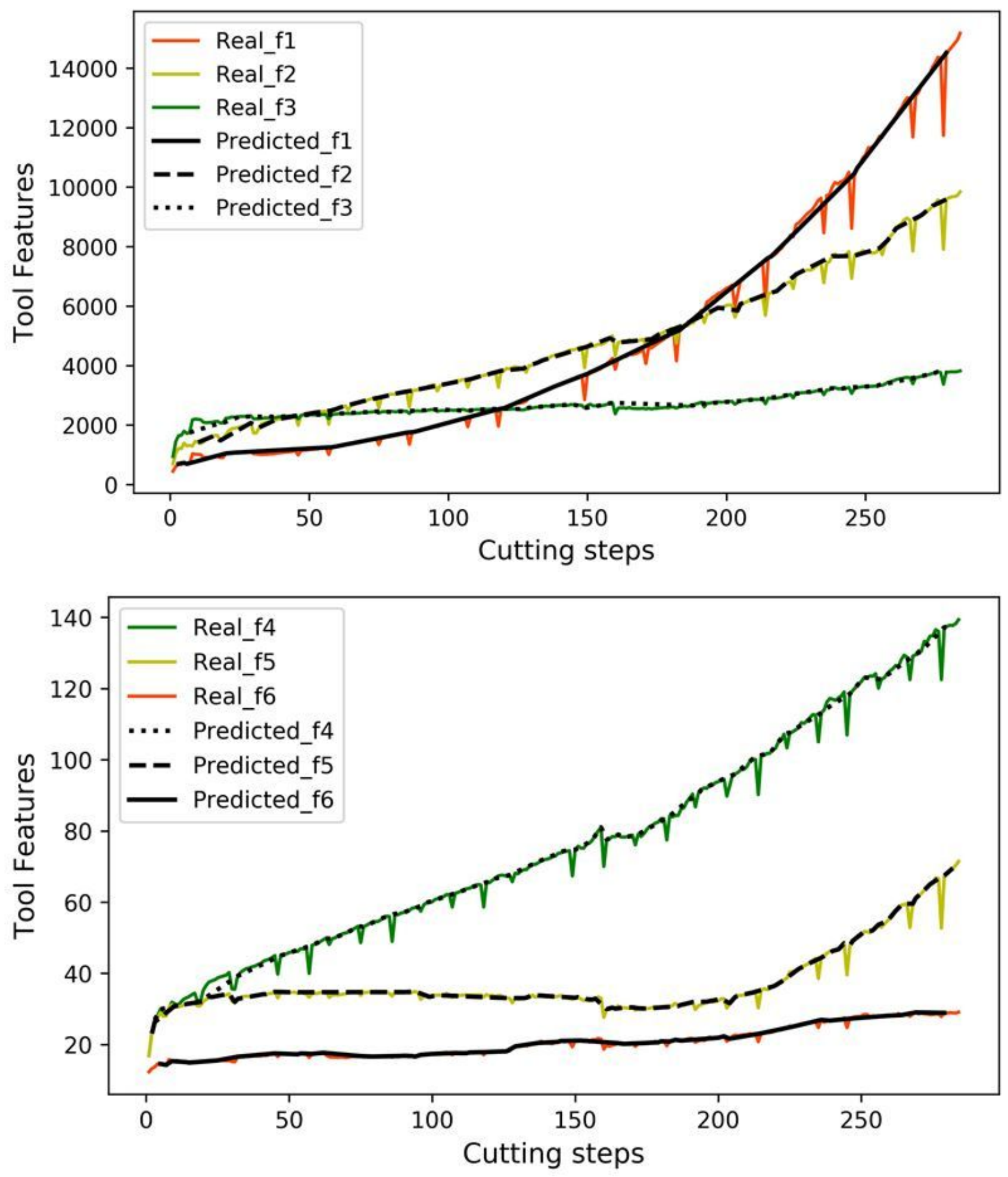

Figure 4

Predicted features of tool2 learned by the DTRL network 


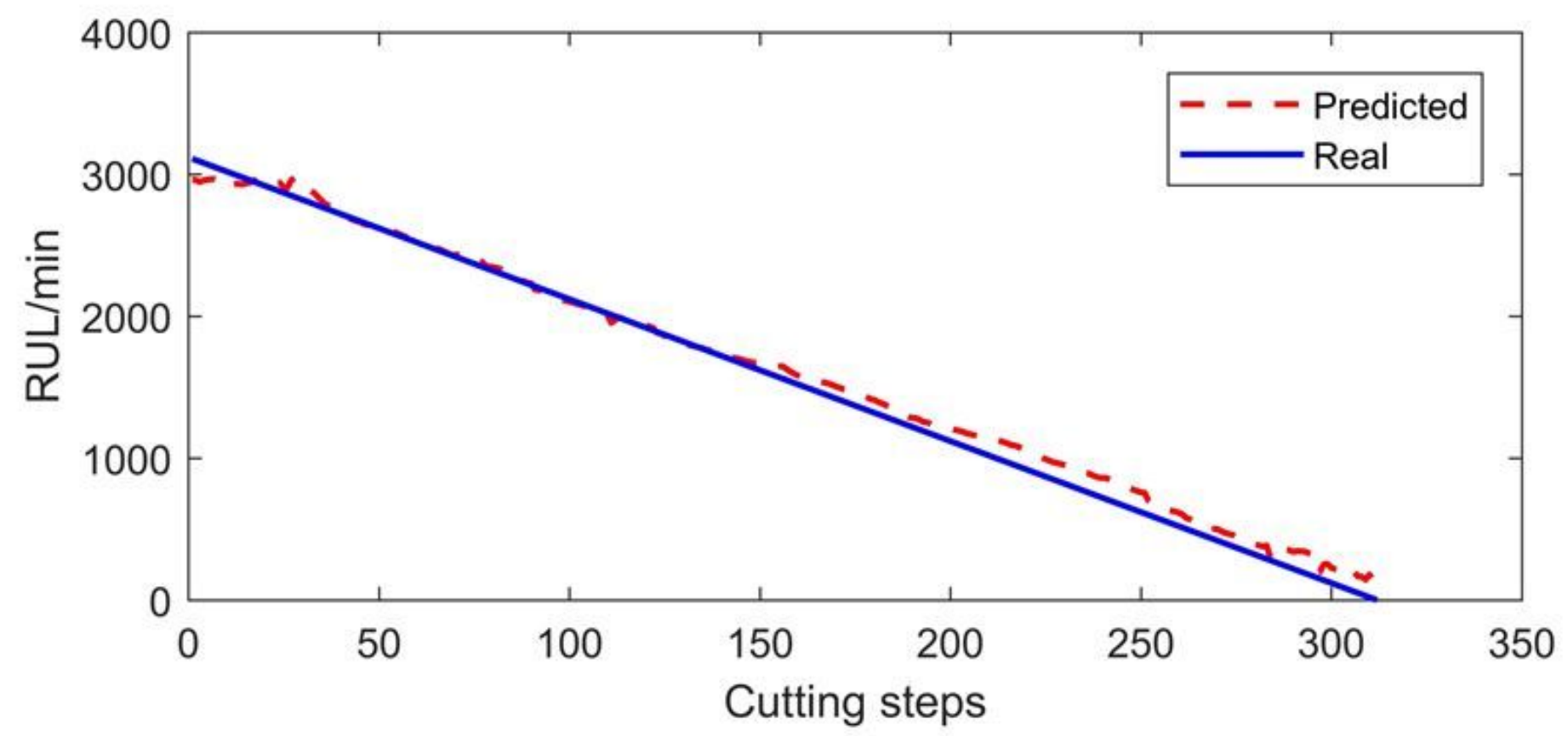

Figure 5

RUL Prediction result for tool2

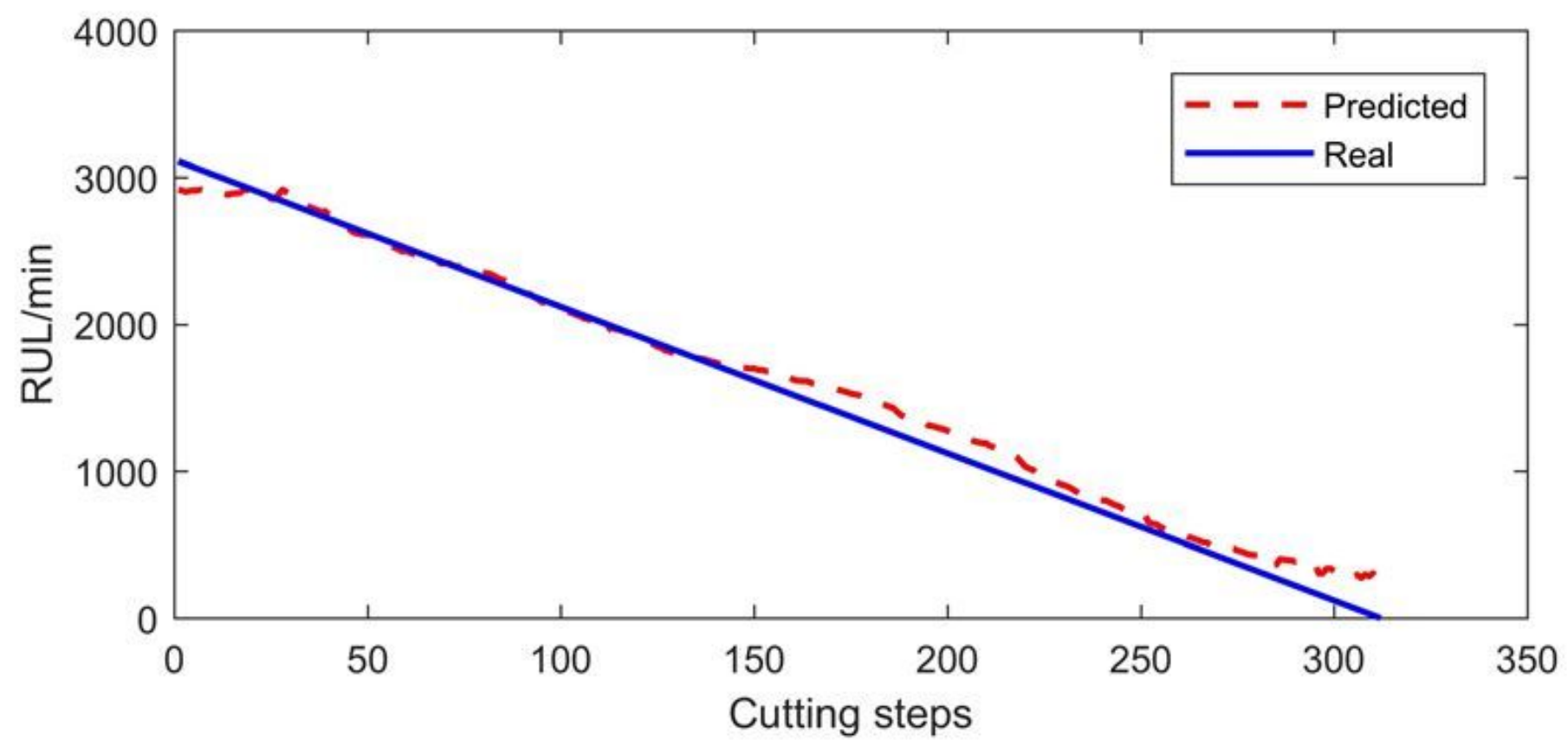

Figure 6

RUL Prediction result for tool2 without deep Q transfer learning 